\title{
Etapas do tratamento de dentes com pino de fibra de vidro: uma revisão de literatura
}

\author{
Steps in tooth treatment with fiberglass post: a literature review \\ Pasos en el tratamiento dental con poste de fibra de vidrio: revisión de la literatura
}

Recebido: 20/10/2021 | Revisado: 27/10/2021 | Aceito: 01/11/2021 | Publicado: 02/11/2021

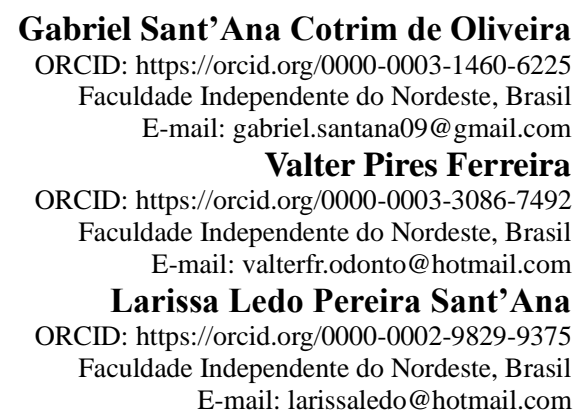

\begin{abstract}
Resumo
Introdução: O pino de fibra de vidro é um material retentor utilizado para o suporte de restaurações ou próteses, que não causa estresse ou fratura à estrutura dentária. Seus benefícios vão desde as propriedades mecânicas semelhantes aos tecidos dentários, até bons resultados estéticos e longevidade. Desta forma, é uma boa opção para a substituição do pino metálico fundido, principalmente por sua característica adesiva ao cimento resinoso, além de ter uma execução clínica simples e rápida. Objetivo: Analisar, através de uma revisão de literatura narrativa, a estrutura e função do pino de fibra de vidro e explorar seus atributos. Metodologia: Trata-se de uma revisão de literatura, em que as buscas foram realizadas nas bases de dados bibliográficos mundiais como: Pubmed, Scielo, Google Acadêmico, Lilacs e Cocrhane. Foram selecionados artigos e trabalhos publicados entre os anos 2010 e 2021, escritos em Inglês, Português e Espanhol. Resultados: Pinos anatômicos revestidos com resina composta resultaram na maior resistência à fratura em comparação com outros métodos. Conclusão: O pino de fibra de vidro é comumente utilizado na odontologia com o principal objetivo de afixar a restauração coronária, reparando, desta forma, função e forma ao elemento dentário. O uso do retentor intarradicular vem sendo progressivamente difundido, pois se trata de um material com boas características estéticas e mecânicas, ideais ao remanescente dentário, além de dispor de biocompatibilidade.
\end{abstract}

Palavras-chave: Técnica para retentor intrarradicular; Pinos dentários; Estética dentária.

\begin{abstract}
Introduction: The fiberglass post is a retaining material used to support restorations or prostheses, which does not cause stress or fracture to the tooth structure. Its benefits range from mechanical properties similar to dental tissue, to good aesthetic results and longevity. Thus, it is a good option for replacing the cast metal post, mainly due to its adhesive characteristic to resin cement, in addition to having a simple and fast clinical execution. Objective: To analyze, through a narrative literature review, the structure and function of the fiberglass post and explore its attributes. Methodology: This is a literature review, in which searches were performed in worldwide bibliographic databases such as: Pubmed, Scielo, Academic Google, Lilacs and Cocrhane. Articles and works published between 2010 and 2021, written in English, Portuguese and Spanish were selected. Results: Anatomic posts coated with composite resin resulted in the highest fracture resistance compared to other methods. Closure: The fiberglass post is commonly used in dentistry with the main objective of affixing the coronary restoration, repairing, in this way, function and shape to the dental element. The use of the intraradicular retainer has been progressively spread, as it is a material with good aesthetic and mechanical characteristics, ideal for remaining teeth, in addition to having biocompatibility.
\end{abstract}

Keywords: Technique for intraradicular retainer; Dental pins; Dental aesthetics.

\section{Resumen}

Introducción: El poste de fibra de vidrio es un material de retención utilizado para soportar restauraciones o prótesis, que no causa estrés ni fractura en la estructura del diente. Sus beneficios van desde propiedades mecánicas similares al tejido dental, hasta buenos resultados estéticos y longevidad. Por tanto, es una buena opción para la sustitución del poste de metal fundido, principalmente por su característica adhesiva al cemento resinoso, además de tener una ejecución clínica sencilla y rápida. Objetivo: Analizar, a través de una revisión de literatura narrativa, la estructura y 
función del poste de fibra de vidrio y explorar sus atributos. Metodología: Se trata de una revisión de la literatura, en la que se realizaron búsquedas en bases de datos bibliográficas mundiales como: Pubmed, Scielo, Academic Google, Lilacs y Cocrhane. Se seleccionaron artículos y trabajos publicados entre 2010 y 2021 , escritos en inglés, portugués y español. Resultados: Los postes anatómicos recubiertos con resina compuesta dieron como resultado la mayor resistencia a la fractura en comparación con otros métodos. Cierre: El poste de fibra de vidrio se utiliza comúnmente en odontología con el objetivo principal de fijar la restauración coronaria, reparando, de esta manera, función y forma al elemento dental. El uso del retenedor intrarradicular se ha ido extendiendo progresivamente, por ser un material con buenas características estéticas y mecánicas, ideal para los dientes remanentes, además de tener biocompatibilidad.

Palabras clave: Técnica de retenedor intradicular; Alfileres dentales; Estética dental.

\section{Introdução}

A odontologia restauradora evolui constantemente, juntamente com os avanços tecnológicos, aprimorando o tratamento e melhorando a qualidade de vida do paciente. A reabilitação funcional e estética dos dentes com tratamento endodôntico, com grande perda de estrutura coronária, recorre-se aos retentores intrarradiculares, com a finalidade da retenção e estabilização do material restaurador (Leal et al, 2018).

O pino de fibra de vidro é um dispositivo, de formato variado, composto de filamentos de fibra de vidro condensados, utilizado em situações de destruição coronária severa ocasionada por lesões cariosas extensas, fraturas dentárias, amplas restaurações e tratamento endodôntico associado ou não a elementos protéticos. Tem como vantagens a sua translucidez, estética, biocompatibilidade, módulo de elasticidade similar à dentina, distribuição homogênea de cargas mastigatórias atuantes na raiz dentária, osso e periodonto, além de conferirem a retenção do conjunto elemento dentário, restauração e pino (Andrioli et al, 2015).

Sua indicação será feita de acordo com alguns critérios observados durante o exame clínico, como a localização do dente na arcada, em razão de que o incisivo, o canino e o pré-molar recebem carga lateral ou de cisalhamento. Já o molar, recebe carga vertical e possui grande quantidade de estrutura dentária, desta forma, o pino de fibra de vidro é menos indicado (Costa et al, 2011).

Previamente à inserção do pino de fibra de vidro, deve-se realizar o tratamento endodôntico no elemento dentário em questão, com o objetivo de acomodar o pino com estabilidade, para que ele receba as forças oclusais de maneira correta. O preparo do conduto radicular deve respeitar as dimensões ideais, de forma que, se tenha disponível 2/3 da raiz em comprimento e 1/3 da raiz em largura. Há casos em que ocorre mutilação intracanal, pois as paredes radiculares podem estar fragilizadas de acordo com o preparo realizado (Caiado et al, 2015).

Apesar das vantagens do pino de fibra de vidro, dentes com canais elípticos apresentam uma inadequação do pino, de forma que a cimentação se torna exagerada, devido à sua desadaptação anatômica ao tipo de canal, o qual pode apresentar bolhas e favorecer a sua descimentação. Para esses casos, existe uma opção de reanatomização do pino utilizando resina composta, desta forma, aumentando a sua adaptação ao conduto e reduzindo a linha de cimento (Freitas et al, 2019).

Para que o tratamento com pino de fibra de vidro seja bem-sucedido é necessário utilizar um material de adesão de partículas orgânicas com partículas inorgânicas, o silano. Este tem propriedades que ligam o material (pino) à dentina (material orgânico). Porém, não é uma ligação considerada forte, pelo pino possuir compósitos que o silano não tem capacidade de unir. (Valdivia et al, 2014).

\section{Metodologia}

O objetivo do presente estudo foi realizar uma revisão de literatura de cunho narrativo e qualitativo, acerca das etapas do tratamento de dentes com pino de fibra de vidro. Uma busca eletrônica foi realizada por meio das bases de dados Pubmed, 
Scielo, Lilacs, Google Acadêmico e Cocrhane, utilizando como descritores em saúde (DeCS): "técnica para retentor intrarradicular; pinos dentários; estética dentária". Foi utilizado o operador lógico booleano "AND" durante a pesquisa.

Foram incluídos artigos de pesquisas e revisões de literatura publicada entre os anos de 2010 até 2021, nos idiomas português, inglês e espanhol, além de dissertações, monografias, teses e pesquisas em livros. Como critérios de escolha, foram selecionados artigos a partir da leitura dos títulos, resumos e posteriormente, o texto.

3. Resultados e DiscussãoO retentor intrarradicular é um dispositivo comumente utilizado em situações de reparação da função de dentes tratados endodonticamente e/ou com comprometimento estrutural, sendo de fundamental importância a seleção correta do pino, pois, este fator pode influenciar na longevidade do tratamento. O pino de fibra de vidro é um dispositivo confeccionado em filamentos de fibra de vidro condensados, sendo uma estrutura sólida, com resistência mecânica e à corrosão, além de ser compatível com a estrutura dentária (Pegoraro et al, 2013).

A forma e o comprimento das raízes são aspectos determinantes para o sucesso do procedimento. Em raízes elípticas e curtas, se faz uso pinos de menor comprimento, já em raízes longas e retas, utilizam-se pinos de maior comprimento, obtendo melhor distribuição de forças e retenção (Barbosa et al, 2016).

A largura do pino deve ser levada em consideração para que ocorra a redução de chances de perfuração e que possa permitir que o dente restaurado resista a fraturas, desta forma, mantém-se a conservação da estrutura dentária (Melo et al, 2015). As proporções de largura devem ser seguidas criteriosamente, de modo que, na porção mais estreita da raiz, a largura do pino deve ser menor que $1 / 3$ da largura da raiz, e deve estar circundado por não menos que $1 \mathrm{~mm}$ de dentina saudável (Ferreira et al, 2018).

A sequência clínica para a implantação do pino de fibra de vidro é simples, porém, deve ser seguida criteriosamente, sem que o cirurgião-dentista seja negligente em qualquer momento. O primeiro passo a ser dado para a aplicação do pino de fibra de vidro, é a realização do preparo radicular (Jha et al, 2012). Esta etapa consiste na remoção parcial do material obturador com o auxílio de brocas pré-fabricadas de diâmetro adaptável ao pino a ser empregado, porém, o mau uso destas brocas pode gerar um desgaste excessivo de dentina (Cardenas et al, 2017).

A desobstrução do canal radicular inicia-se com a medida do dente através da radiografia e definição da quantidade de Guta-Percha que será removida. O pino de fibra de vidro deve abranger $2 / 3$ do remanescente dentário, mantendo cerca de $3 \mathrm{~mm}$ a 5mm de material obturador. A próxima etapa da sequência clínica consiste na escolha pino (Muniz et al, 2010).

A opção correta é aquela que permite a preservação de maior quantidade de dentina no preparo, deste modo, tem-se no mercado, pinos de fibra de vidro com diferentes conicidades, acompanhados por alargadores, visando uma melhor acomodação às paredes do conduto. A seleção é feita com pino sobreposto à radiografia, mantendo-se preservado cerca de $4 \mathrm{~mm}$ de Guta-Percha e o diâmetro escolhido é aquele que fique mais próximo da luz do canal (Muniz et al, 2011).

O pino de fibra de vidro, do mesmo modo que qualquer outro núcleo intrarradicular, necessita de um tratamento prévio com a finalidade de aderir o material às paredes do conduto de maneira eficaz, auxiliando, desta forma, na retenção micromecânica e química entre o conduto e o pino. O material utilizado para o tratamento é o silano, que possui características orgânicas e inorgânicas capazes de possibilitar a adesão química entre a dentina e o pino (Valdivia et al, 2018).

No tratamento micromecânico, é utilizado o ácido fosfórico, como agente de limpeza, ácido fluorídrico e peróxido de hidrogênio, com o intuito de formar porosidades por toda a superfície do pino. No tratamento de superfície realizado em escala industrial, são dispensadas as etapas clínicas, pois se faz uso do cimento autoadesivo, que também isentam o tratamento prévio do substrato dental (Marques et al, 2016).

No mercado atual, existem os cimentos autoadesivos criados com o objetivo de superar as deficiências dos cimentos resinoso e convencionais, tais como, interferência de adesão na presença do Eugenol e sensibilidade. De acordo com seu 
fabricante, não se tem riscos de sensibilidade pós-operatória, uma vez que, não se remove smear layer. Além disso, o cimento autoadesivo contém adesão semelhante aos cimentos resinosos, boas propriedades mecânicas, simplificação da técnica, estabilidade dimensional e estética favorável (Magalhães et al, 2018).

\subsection{Anatomia do conduto radicular e Técnica terapêutica}

Pegoraro et al., (2014), afirma que, quando o conduto se apresenta muito alargado ou ovalado, especialmente nos terços médio e cervical, as superfícies do pino não se adaptam a suas paredes. Para evitar espessuras exageradas de cimento em algumas áreas e o consequente aumento da contração volumétrica de polimerização, do estresse na interface cimento/dentina e da presença de bolhas com diminuição da resistência do cimento, faz-se o reembasamento do pino com resina composta no conduto, técnica conhecida como pino anatômico.

Soares et al., (2018), acredita que um pino de fibra de vidro de geometria padrão deixa espaço em excesso dentro do canal radicular alargado para ser preenchido com uma grande quantidade de material de cimentação. Isso resulta em uma área potencialmente fraca, o que pode comprometer o prognóstico em longo prazo, desta forma, um método alternativo simples é realinhar os pinos de fibra de vidro com resina composta. Esse pino anatômico reduz o volume do cimento de cimentação e se adapta melhor ao canal parede. Pinos anatômicos revestidos com resina composta resultaram na maior resistência à fratura em comparação com outros métodos.

Valdivia et al., (2018), considera que a conservação da estrutura dentária é o parâmetro mais importante para melhorar o comportamento biomecânico dos dentes tratados endodonticamente e reabilitados com pinos de fibra de vidro. Estudos têm demonstrado que a manutenção de 1,5 a $2 \mathrm{~mm}$ melhora as distribuições de tensões na dentina radicular e ao longo da interface pós-dentina.

\section{Conclusão}

O pino de fibra de vidro é comumente utilizado na odontologia com o principal objetivo de afixar a restauração coronária, reparando, desta forma, função e forma ao elemento dentário. O uso do retentor intarradicular vem sendo progressivamente difundido, pois se trata de um material com boas características mecânicas e estéticas, ideais ao remanescente dentário, além de dispor de biocompatibilidade.

Desta forma, é de suma importância o conhecimento a cerca do tratamento do pino de fibra de vidro, bem como, o material a ser empregado e as características gerais do remanescente dentário, pois são cruciais para o êxito do tratamento. Qualquer imprecisão na execução clínica pode acarretar em insucesso, diante disto, é indispensável a sequência clínica de forma minudente.

Assim, espera-se que esta revisão de literatura possa contribuir com o melhor entendimento a respeito dos aspectos e etapas do tratamento com pino de fibra de vidro, e que possa desta forma, impulsionar as futuras pesquisas da área.

\section{Referências}

Andrioli, A. R. V., Coutinho, M., Vasconcellos, A. A. de, \& Miranda, M. E. (2016). Relining effects on the push-out shear bond strength of glass fiber posts. Revista de Odontologia Da UNESP, 45, 227-233. https://doi.org/10.1590/1807-2577.14815.

Barbosa, I. F., Barreto, B. C. T., Coelho, M. D. O., Pereira, G. D. D. S., \& Carvalho, Z. M. C. D. (2016). Pinos de Fibra: Revisão da Literatura. Revista Uningá Review, 28(1).

Caiado, A. C. R. L., de Goes, M. F., de Souza-Filho, F. J., \& Rueggeberg, F. A. (2010). The effect of acid etchant type and dentin location on tubular density and dimension. The Journal of Prosthetic Dentistry, 103(6), 352-361. https://doi.org/10.1016/s0022-3913(10)60076-5.

Cardenas, J. E. V. (n.d.). Avaliação da espessura de cimento e resistência adesiva de pinos de fibra de vidro cônicos em preparo para retentor intra-radicular realizados com uma ponta ultrassônica desenvolvida. https://doi.org/10.11606/d.23.2018.tde-24012018-114059. 
Costa, D. D., Carvalho, L. C. B., Nery, F. S., Barbosa, P. A., Amado, D. S., \& Cruz, J. F. W. (2011). Avaliação quantitativa do remanescente dentinário após instalação de pino de fibra de vidro em incisivos inferiores com raízes achatadas. Revista de Ciências Médicas E Biológicas, 10(1), 39. https://doi.org/10.9771/cmbio.v10i1.5227.

Ferreira, G. C., Bueno, M. G., \& Amorim, E. D. (2018). Reabilitação em dentes anteriores com pinos de fibra de vidro e coroas metal free: relato de caso. Revista Da Faculdade de Odontologia - UPF, 23(3), 300-304.

Freitas, T. L. de, Vitti, R. P., Miranda, M. E., \& Brandt, W. C. (2019). Effect of Glass Fiber Post Adaptation on Push-Out Bond Strength to Root Dentin. Brazilian Dental Journal, 30(4), 350-355. https://doi.org/10.1590/0103-6440201902491.

Jha, P., \& Jha, M. (2012). Retention of fiber posts in different dentin regions: An in vitro study. Indian Journal of Dental Research, 23 (3), 337. https://doi.org/10.4103/0970-9290.102219Leal, G. S., Souza, L. T. R., Dias, Y. V., \& Lessa, A. M. G. (2018). Características do Pino de Fibra de Vidro e aplicações Clínicas: Uma Revisão da Literatura. ID on Line Revista De Psicologia, 12(42), 14-26. https://doi.org/10.14295/idonline.v12i42.1413.

Leal, G. S., Souza, L. T. R., Dias, Y. V., \& Lessa, A. M. G. (2018). Características do Pino de Fibra de Vidro e aplicações Clínicas: Uma Revisão da Literatura. ID on Line REVISTA de PSICOLOGIA, 12(42), 14-26. https://doi.org/10.14295/idonline.v12i42.1413.

Magalhães, I. C., Diógenes, M. A. R., Lima, T. H., \& Monteiro, L. K. B. (2018). Uso De Cimentos Convencionais X Cimentos Resinosos Na Cimentação De Pinos De Fibra De Vidro. Jornada Odontológica Dos Acadêmicos Da Católica, 4(1). http://publicacoesacademic as.unicatolicaquixada.edu.br/index.php/joac/article/view/2474.

Marques, J., Carolina, B. G., Silva, E. M. P., Renata, a.s., Maíra, P. (2016). Análise comparativa da resistência de união de um cimento convencional e um cimento autoadesivo após diferentes tratamentos na superfície de pinos de fibra de vidro. Revista de Odontologia da UNESP; 45(2), $121-126$.

Melo, A. R. S. de, Almeida, A. N. C. L. de, Sales, T. L. de L., Madureira, I. T., Figueiroa, A., \& Leite, E. B. da C. (2015). Reconstrução De Dentes Severamente Destruídos Com Pino De Fibra De Vidro. Odontologia Clínico-Científica (Online), 14(3), 725-728. http://revodonto.bvsalud.org/scielo.php?script=sci_arttext\&pid=S1677-38882015000300009.

Muniz, L. (2010). Pinos de fibra: técnicas de preparo e cimentação. Revista Brasil Dentistry Clínica.

Muniz, L. (2011). Reabilitação estética em dentes tratados endodonticamente: Pinos de fibra e possibilidades clínicas conservadoras. Livraria Santos, Editora, Santos $-S P$.

Pegoraro, L. F., Do Valle, A. L., Araújo, C. R. P., Bonfante, G., Conti, P. C. R. (2013). Prótese Fixa: Bases para o planejamento em reabilitação oral. 2. Ed. Artes Médicas. São Paulo - SP.

Pegoraro, L. F. (2014). Fundamentos da prótese fixa. Arte Médicas. São Paulo - SP.

Sá, T. C. M., Akaki, E., \& Sá, J. C. M. (2010). Pinos Estéticos: Qual O Melhor Sistema? Arquivo Brasileiro de Odontologia, 6(3), 179-184. http://periodicos.pucminas.br/index.php/Arquivobrasileirodontologia/article/view/2183.

Soares, C. J., Rodrigues, M. de P., Faria-E-Silva, A. L., Santos-Filho, P. C. F., Veríssimo, C., Kim, H.-C., \& Versluis, A. (2018). How biomechanics can affect the endodontic treated teeth and their restorative procedures? Brazilian Oral Research, 32(suppl 1), e76. https://doi.org/10.1590/1807-3107bor2018.vol32.0076.

Valdivia, A. D. C. M., Novais, V. R., Menezes, M., Roscoe, M. G., Estrela C., \& Soares, C. J. (2014). Effect of Sufarce Treatment of Fiberglass Posts on Bond Strength to Root Dentin. Brazilian Dental Journal. 25(4), 314-320.

Valdivia, A. D. C. M., Rodrigues, M. de P., Bicalho, A. A., Van Meerbeek, B., Sloten, J. V., Pessoa, R. S. E., \& Soares, C. J. (2018). Biomechanical Effect of Ferrule on Incisors Restored with a Fiberglass Post and Lithium-Disilicate Ceramic Crown after Thermal Cycling and Fatigue Loading. The Journal of Adhesive Dentistry, 20(2), 133-142. https://doi.org/10.3290/j.jad.a40305. 